\title{
Resultados tardios das reconstruções arteriais dos membros inferiores com a utilização de veias portadoras de dilatações varicosas revestidas seletivamente com segmentos protéticos
}

\author{
Late results of infrainguinal arterial bypasses using dilated varicose veins \\ selectively wrapped with prosthetic segments
}

\author{
Didier M ellière'1, M aria Claudia de Albuquerque², Pascal D esgranges, \\ Eric Allaire, Jean Pierre Becquemin ${ }^{5}$
}

\section{Resumo}

O bjetivo: Responder as seguintes questões: 1) o revestimento protético deve ser precedido de uma redução da ectasia? 2) as zonas revestidas apresentam um risco de hiperplasia ou estenose? 3) as zonas não revestidas possuem um risco de dilatação e ruptura?

M étodos: D oze pacientes ( 10 homense duas mulheres), com idades entre 33 e 77 anos (idade mediana $=68$ ), foram submetidos a pontes em conseqüência de arterite $(n=7)$, aneurisma poplíteo $(n=4)$ ou ruptura de prótese $(n=1)$. As pontes foram fêmoro-poplíteas $(n=8)$, fêmoro-infra poplíteas ( $n=3$ ) e poplíteo-poplítea $(n=1)$. A safena utilizada foi de forma reversa em nove casos eex situ devalvuladas em três casos. O s revestimentos foram aplicados em número de um $(n=2)$, dois $(n=3)$, três $(n=6)$ e quatro $(n=1)$. Todos, exceto um, foram de PTFE.

Resultados: Duas pontes ocluíram, uma precocemente por déficit de leito distal e outra 4 anos após a operação. As outras 10 pontes permaneceram pérvias durante 0 acompanhamento, que variou entre 1 e 11 anos (mediana $=4$ anos). M etade dos casos apresentou uma deterioração progressiva do leito distal. N o último controle, duas pontes permaneceram pérvias, a despeito de um leito distal desértico.

C onclusão: 0 s resultados desta série apresentam-se notadamente superiores aos das pontes protéicas descritas na literatura. 0 revestimento é efetuado facilmente com uma prótese curta de PT FE de parede fina. É inútil reduzir as dilatações antes do revestimento por sutura ou ressecção - anastomose. As zonas situadas sobre a bainha de PTFE não evoluem para estenoses. As zonas intermediárias dilatamse por vezes moderadamente e sem risco de ruptura.

Palavras-chaves: pontes arteriais, enxertos, veias varicosas.

\begin{abstract}
O bjective: To answer the following questions: 1) is it necessary to excise and suture the dilatations before wrapping? 2) are the wrapped segments at risk of hyperplasia or stenosis? 3) are the non-wrapped areas at risk of dilatation and rupture?

Methods: Infrainguinal revascularization was performed in 12 patients (10 males, two females), aged 33-77 years (mean age $=68$ ). Surgical indication was arteritis $(n=7)$, popliteal aneurysm $(n=4)$, or rupture of $\mathrm{aD}$ acron graft $(n=1)$. Location of the bypass was femoropopliteal $(n=8)$, femoro-infrapopliteal $(n=3)$ or popliteo-popliteal $(n=1)$. Position of the vein was ex situ, either reversed $(n=9)$ or nonreversed devalvulated $(n=3)$. The number of dilatations reinforced with a graft were one $(n=2)$, two $(n=3)$, three $(n=6)$ and four $(n=$ 1). All reinforcements except one were made with PTFE grafts.

Results: Two vein bypasses occluded, one early due to distal bed deficit and the other one 4 years after the surgery. The other 10 grafts remained patent during the follow-up, which ranged from 1 to 11 years (mean $=4$ years). $\mathrm{H}$ alf of the patients demonstrated some degree of progressive deterioration of the distal bed. At the last control, two patients had a patent bypass in spite of a deserted run-off.

C onclusion: The results of this series show that long-term patency of the wrapped vein-bypasses look far better than those of prostheticgrafts in this location described in the literature. The wrapping can be easily performed with a short thin wall PTFE graft. It is useless to reduce the dilatations before the wrapping by suture or resection anastomosis. Wrapped segments will not devel op hyperplastic stenosis. Unwrapped segments may enlarge moderately without a risk of rupture.
\end{abstract}

Key words: arterial bypass, grafts, varicose veins.

1. Professor, Centro Hospitalar Universitário Henri Mondor, Cretéil, França.

2. Residente, Serviço de Cirurgia Vascular, Centro Hospitalar Universitário, Hospital Henri Mondor, Cretéil, França.

3. Professor, Cirurgia Vascular, Centro Hospitalar Universitário Henri Mondor, Cretéil, França.

4. Cirurgião vascular, Centro Hospitalar Universitário Henri Mondor, Cretéil, França.

5. Professor e chefe do Serviço de Cirurgia Vascular, Centro Hospitalar Universitário Henri Mondor, Cretéil, França.

Artigo submetido em 13.07.05, aceito em 21.10.05. 
Existe um consenso, dentro de todas as indicações de pontes infra-inguinais, sobre a superioridade da utilização de veias em relação àqueles que utilizam próteses, o que se torna mais importante quanto mais distal for a anastomose. D istal: sobre a artéria poplítea, abaixo do joelho, artérias da perna ou do tornozelo. As taxas médias de perviedade em 5 anos das pontes realizadasparatratamento declaudicação intermitente, segundo o TransAtlantic Inter-Society Consensus $\left(\right.$ TASC $^{1}$, são de $80 \%$ para as realizadas com veias, seja qual for o nível; $75 \%$ com PTFE acimado joel ho e $65 \%$ com PTFE abaixo do joelho; nos casos de pontes fêmoro-poplíteas por isquemia crítica, essas taxas em meta-análise são de 66, 47 e 33\%, respectivamente. A recomendação 92 estipula que "a safena interna adequada éo conduto ideal para as pontesfêmoro-poplíteas abaixo do joel ho e distais. $N$ a sua ausência, é preciso utilizar uma outra veia de boa qualidade". D a mesma forma, os Repères pour les D écisions en Chirurgie V asculaire, editados pel o Colégio Francês de Cirurgia V ascu$\operatorname{lar}^{2}$, no capítulo “Estenose ou oclusão isolada da artéria femoral superficial no estado de claudicação intermitente, versão 2000", indica que diferentes estudos demonstram a superioridade das pontes venosas. $\mathrm{Na}$ ausência de safena disponível, numerosos autores mostraram a importância das pontes realizadas, seja com veias dos braços ${ }^{3,4}$ e/ou com segmentos de veias anastomosados entre $\mathrm{si}^{5}$.

$\mathrm{N}$ a prática, os cirurgiões vasculares deparam-se freqüentemente com a falta de veias adequadas em diversas situações: retirada anterior das veias para tratamento de varizes, exérese de veias para outras pontes, paredes fibrosas, diâmetro insuficiente ou presença de varizes. N este último caso, não é raro que a única veia utilizável apresente uma ou múltiplas dilatações isoladas, embora os diâmetros dossegmentosintermediários sejam adequados. Existem várias razões para rejeitar essas veias: risco deruptura, risco associado a ressecçõesanastomoses múltiplas, risco de estenose no caso de redução por sutura simples. Ao contrário, seria lícito utilizar uma veia cujas dilatações localizadas poderiam ser revestidas seletivamente por curtos segmentos de próteses? Em estudos anteriores, obteve-se resultados precoces satisfatórios com a utilização de veias revestidas, umas sel etivamente e outras na total idade ${ }^{6}$. Posteriormente, foi constatado que, quando as dilatações venosas são localizadas, o revestimento seletivo parece proporcionar resultados em curto prazo também mais favoráveis que o revestimento total, pois além de ser de execução mais fácil, existe menor risco de lesão da veia na introdução em um curto segmento de prótese.

$\mathrm{N}$ este estudo, o resultado das pontes efetuadas pelo primeiro autor com veias cujas dilatações tivessem sido revestidas por um curto segmento de prótese foi avaliado, a fim de responder as seguintes questões: o revestimento deve ser precedido de uma redução da ectasia? A zona recoberta evolui para hiperplasia e estenose? As zonas não recobertas evoluem para dilatação eruptura?

\section{Pacientes e métodos}

Foi feito um estudo retrospectivo descritivo de pequena série de casos. Entre 1992 e 2001, a técnica de revestimento sel etivo foi utilizada em 12 pacientes, sendo 10 homens e duas mulheres, com idades entre 36 e 71 anos (idade mediana = 68 anos), os quais necessitavam de pontes infra-inguinais. 0 ito deles tinham uma arteriopatia obliterante: seis sofriam de claudicação intermitente invalidante e resistente ao tratamento médico clássico, um apresentava dor de repouso (estado III da classificação de Leriche e F ontaine) e o último apresentava escaras no calcanhar e dedos dos pés. D ois deles apresentavam uma oclusão da artéria femoral associada e um aneurisma poplíteo inicial. Somente um tinha um leito distal com deságüe considerado normal: quatro tinham duas artérias da perna ocluídas, mas guardavam um eixo comunicante ao arco plantar; três apresentavam uma ou duas artérias que se ocluíram progressivamente; no último paciente, o leito distal era reduzido a algumas finas colaterais, que nasciam do tronco tíbio-peroneiro. Os antecedentes desses pacientes comportavam doenças habituais. 0 paciente de 36 anos com isquemia crítica tinha um antecedente familiar de arterite e tabagismo (duas carteiras/dia) associado.

T rês outros pacientes eram portadores de aneurisma poplíteo: um era assintomático, mas a arteriografia mostrava oclusão da artéria tibial anterior; um tinha o aneurisma com trombose parcial e seu leito distal era limitado a uma artéria peroneira; o último tinha previamente uma ponte com veia que ocluiu, gerando uma isquemia aguda. Este foi submetido a trombólise, porém evoluiu com persistência de numerosos coágulos. A presentava um aneurisma poplíteo contral ateral associado, com indicação cirúrgica pelas suas dimensões. Finalmente, um pacientemultioperado por arteriteteve um falso aneurisma associado na altura do triângulo de 
Scarpa e uma ruptura de uma prótese fêmoro-poplítea acima do joelho em três locais, com um leito distal reduzido somente à artéria peroneira.

As pontes foram fêmoro-poplíteas $(n=8)$, fêmoroartérias da perna $(n=3)$ e poplíteo-poplítea $(n=1)$. As condições anatômicas favoreceram a utilização da veia safena reversa em nove casos e ex situ devalvuladas em três casos. Em um paciente, uma ponte foi realizada com veias do braço (radial + cefálica) previamente dilatadas por criação deumafístula artério-venosa. Para todos os outros, a veia utilizada foi a safena magna.

As técnicas de dissecção da safena eas anastomoses não tiveram particularidades. 0 diâmetro da veia variou entre 4 e $9 \mathrm{~mm}$, apresentando $5 \mathrm{~mm}$ em cinco casos. As veias foram introduzidas dentro do revestimento protético e posicionadas fora da zona de dobra do joelho. Esses revestimentos foram realizados apenas uma vez com segmento de D acron ( $C$ ardial, F rança), sete vezes com PTFE habitual e, nos últimos quatro casos, com um tubo de PTFE de parede fina de $6 \mathrm{~mm}$, destinado a pontes carotidianas $\left(\mathrm{G}\right.$ ore $\left.{ }^{\circledR}\right)$. 0 comprimento do revestimento variou de 2 a $6 \mathrm{~cm}$, e a quantidade utilizada para a mesma veia foi de um $(n=2)$, dois $(n=3)$, três $(n=6)$ ou quatro $(n=1)$. A fim de evitar o deslocamento do revestimento sobre a veia durante sua passagem no interior do tunelizador, uma ou as duas extremidades foram fixadas à sua adventícia por um ponto com fio 7.0. 0 êxito do procedimento foi observado na arteriografia transoperatória. Todos os pacientes operados foram examinados 1 mês após a operação e 1 ano mais tarde com a avaliação do ecoD oppler. Em seguida, os pacientes foram orientados a efetuar, a cada ano, um controle com eco-D oppler e a se consultar em caso de problemas clínicos ou detectados à ultra-sonografia. Os pacientes foram contatados para o estudo e solicitados a se consultar ou a trazer um último exame de eco-D oppler (o pequeno número de pacientes não autoriza a utilização de testes estatísticos) (Figuras 1 e 2).

\section{Resultados}

A ponte realizada para salvamento do membro de um homem de 36 anos ocluiu rapidamente. Esse paciente foi amputado. A anastomose inferior foi realizada sobre a artéria poplítea terminal, apesar de bastante infiltrada. A arteriografia transoperatória mostrou que o tronco tíbio-peroneiro estava reduzido a uma luz filiforme. Por isso, descemos um guia até o interior da artéria tibial anterior edilatamos o troco tíbio-peronei-



Figura 1 - Ponte com veia apresentando quatro pequenas dilatações. A anastomose superior foi concluída. D uas dilatações foram recobertas, e as outras duas são indicadas distalmente pela pinça de dissecção

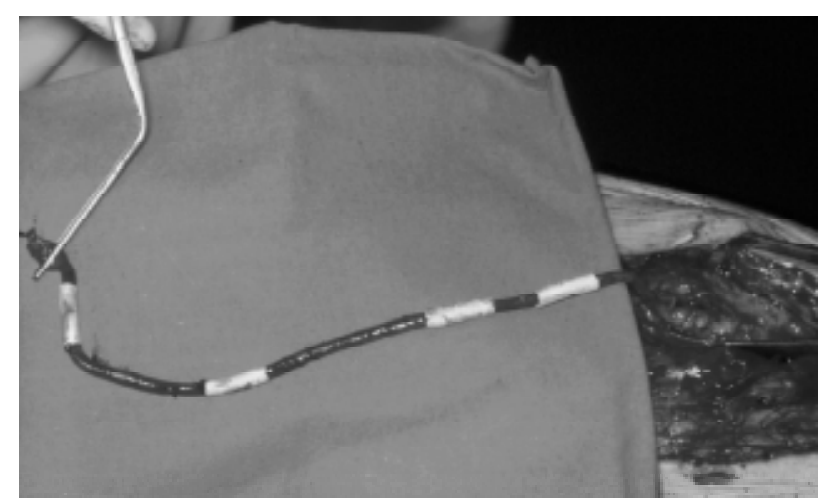

Figura 2 - As quatro dilataç̃ões foram recobertas

ro com um balão de $3 \mathrm{~mm}$. N ão foi possível, no entanto, descer 0 balão à artéria tibial anterior. A oclusão da ponte deveu-se à deficiência de um leito distal, enão à técnica de revestimento.

As 11 pontes restantes foram seguidas durante um período de 13 a 133 meses, com a média de 47 meses. Três dos operados morreram de causas intercorrentes, dois após 1 ano de cirurgia e um após 2 anos, com as pontes pérvias. Os oito sobreviventes foram seguidos, respectivamente, por 1 ano $(n=1), 4$ anos $(n=4), 5$ anos $(n=1), 6$ anos $(n=1)$ e 11 anos $(n=1)$. U ma ponte ocluiu após 4 anos, o que levou à amputação da perna (tratava-se de uma ponte realizada em um paciente já operado de revascularização do membro inferior para correção de aneurisma poplíteo; sua primeira ponte trombosou efoi parcialmente repermeabilizada através da fibrinólise, mas deixou numerosos coágulos no leito 
distal). Todas as outras permaneceram pérvias. U ma delas apresentou uma estenose da anastomose proximal 6 meses após a cirurgia, a qual foi tratada pela interposição de um curto segmento protético, não tendo relação com a veia revestida. 0 utra pontedilatou-seglobalmente, em média $1 \mathrm{~mm}$.

$\mathrm{N}$ ão foram observadas nem estenoses do segmento recoberto, nem dilatações significativas das zonas intermediárias, masa metadedosoperados desenvolveu uma deterioração progressiva do leito distal. No último controle, duas das pontes pérvias apresentavam um leito distal desértico.

\section{D iscussão}

A primeira ponte longa com veia safena invertida foi efetuadapor J. Kunlin em 1948, utilizando uma veia que apresentava dilatação varicosa; ficou pérvia até a mortedo paciente, 28 anosmaistarde. A ectasia permaneceu estável e foi tratada por ressecção - sutura, 14 anos após, no momento da aposição de uma prótese aorto-bifemoral. Aos 20 anos, a ponte venosa dilatouse, em média, 1/3 de seu diâmetro; aos 27 anos, era portadora de placas ateromatosas palpadas durante uma reintervenção para correção de falso aneurisma da anastomose superior ${ }^{7}$.

A dilatação an eurismática das pontesvenosaséuma complicação rara8-13, que pode evoluir para uma ruptura ${ }^{12,13}$. Em nossa experiência, quando a dilatação é detectada a tempo, sempre pode ser tratada por substituição completa, se toda a veia estiver dilatada, ou por substituição parcial ou simples revestimento externo, se a dilatação for segmentar. 0 prazo médio para um desenvolvimento de dilatação é de 7 anos ${ }^{8}$. As causas são ainda mal elucidadas. Cogita-se serem secundárias a ateromas 9,11 (apesar de os tecidos dessas dilatações não diferirem freqüentemente do resto da ponte ${ }^{14}$ ), ao tabagismo eà existência de uma doença aneurismática. L oftus et al. constataram, em um estudo prospectivo, 0 desenvolvimento de $42 \%$ de aneurismas venosos nas pontes para correção de aneurisma da artéria poplítea versus $2 \%$ naqueles por isquemia crônica ${ }^{10}$. Poderíamostemer particularmenteessa evolução quan do a veia utilizada para a ponte apresenta uma ou múltiplas varizes cuja parede habitualmente se afina, podendo levar a uma fragilidade de toda a parede venosa. $\mathrm{N} 0$ entanto, nunca observamosessa complicação, quetambém não foi constatada em outros três estudos: no de $M$ oritz et al., que utilizaram nove revestimentos curtos e parciais ${ }^{15}$, no estudo de Soury et al., que tiveram somente um caso ${ }^{16}$, eno de $\mathrm{N}$ eufang et al., que estudaram a evolução de 35 pontes, durante uma média de 28 meses, sendo todos os pacientes portadores de varizes tratadas por revestimentos curtos de PT FE ${ }^{17}$.

O utra complicação potencial seria o desenvolvimento de uma estenose hiperplásica ao nível das zonas recobertas. $\mathrm{N}$ o seu estudo experimental em carneiros, $M$ oritz et al. ${ }^{15}$ não observaram estenoses. Ao contrário, comparando pontes fêmoro-poplíteas efetuadas com veias femorais e outras com as quais veias jugulares foram previamente introduzidas em uma rede tubular de largos poros de $D$ acron, constataram que havia uma hiperplasia menos importante no segundo grupo. Os mesmos autores, em uma série de 19 pontes em humanos, observaram somenteum caso deestenoseno enxerto em uma zona não recoberta pela rede. T ambém não foi constatado por Soury et al. ${ }^{16}, \mathrm{~N}$ eufang et al. ${ }^{17}$, nem por nós. A arterialização da veia acarreta sempre um certo grau de hiperplasia. Parece que 0 revestimento total por uma rede de $D$ acron diminuiria a hiperplasia, por reduzir as forças oponentes à parede venosa ${ }^{15}$. $M$ oritz et al. constataram um aumento da densidade da vasa vasorum sobre a rede de $D$ acron. Em um estudo anteriormente feito em animais, Karayannacos et al. mostraram quea porosidade da próteseéessencial, pois um espessamento parietal apareceu quando a prótese não era porosa ${ }^{18}$. N o entanto, como N eufang et al. ${ }^{17}$, utilizamos PT FE e não observamos estenoses, possivelmente porque os segmentos recobertos foram suficientemente curtos para não impedir o desenvolvimento da vasa vasorum.

Podemos criticar nosso estudo pela brevidade do acompanhamento médio, que foi de um pouco menos de 4 anos, e por um pequeno número de casos. Em relação à duração da observação, foi suficiente para avaliação do risco de hiperplasia, pois na maioria das vezes se desenvolve durante o primeiro ano. $\mathrm{N}$ o entanto, foi muito curto para análiseda degeneração aneurismática, mas se essa complicação viesse a aparecer, poderia ser facilmentedescoberta por um exameclínico e um eco-D oppler periódicos. A correção é fácil e eventualmente sob anestesia local. Q uanto ao pequeno número de casos, compensa-se pelo fato de que nossa observação se adiciona àquela de $\mathrm{N}$ eufang et al., cujo estudo comportou o triplo do nosso. Em seu estudo17, as perviedades primária e secundária assistidas em 4 anos das pontes com veias revestidas foram, respectivamente, de 66 e $82 \%$. Esses resultados parecem ser notadamente superiores àqueles das pontes protéticas 
descritas na literatura. I sso se explica, provavelmente, pela conservação da complacência parietal e da antitrombogenicidade da íntima.

Seis detalhes merecem atenção: 1) é inútil ressecar ou reduzir por sutura as dilatações, contrariamente ao que foi proposto por outros autores ${ }^{16}$; o tecido excedente apoia-se sobre o revestimento protético sem problemas; $M$ oritz et al. ${ }^{15}$ constataram que podemosreduzir, sem complicações, o diâmetro da veia em $50 \%$ com o revestimento; 2) ao nível do revestimento, a origem das grandes colaterais deve ser ressecada, a fim deevitar a formação deuma sal iência no interior daluz, enquanto as pequenas colaterais devem ser ligadas com um fio fino; 3) a veia deve ser posicionada de maneira que 0 revestimento não fiqueao nível da dobra do joelho, que se situa um pouco acima da interlinha articular; 4) a prótese mais adequada para os revestimentos curtos parece ser o PT FE de parede fina, que podemos facilmentedilatar ao diâmetro desejável com aajuda deuma pequena pinça; para os revestimentos longos, é preferível utilizar um fileteprotético delargosporos, taiscomo ProVena fabricado pela B. Braun Vascular Systems (Bethlehem, PA, EUA); 5) para evitar o deslize do material de revestimento sobre a veia durante a passagem pelo interior do tunelizador, é preciso fixar com um ponto as extremidades à adventícia com fio número 7.0 ; 6) em seguida, éprudentea vigilância dessas pontes anual mente com eco-D oppler durantetoda a vida. Essa técnica também podeser utilizada para as pontes in situ, cortando lateralmente a prótese e suturando-a com alguns pontos separados.

$\mathrm{N}$ a série de $\mathrm{N}$ eufang et al. ${ }^{17}$, sobre um total de 932 pontes infra-inguinais, 3,8\% foram beneficiadas por essa técnica, evitando, dessa forma, uma ponte protética.

\section{Conclusão}

A presença de algumas dilatações sobre uma veia cujo calibre é globalmente adequado não justifica sua rejeição quando não há outra veia disponível. A perviedade dessas veias revestidas é superior à das pontes unicamente protéticas. Essa noção deveria ser conhecida por aqueles que efetuam essa cirurgia, mas também pelos cirurgiões e angiologistas que tratam de varizes.

\section{Referências}

1. Transatlantic Inter-Society Consensus - M anagement of Peripheral Arterial D isease. J V asc Surg. 2000;31:S113-222.
2. CollègeF rançaisdeC hirurgieV asculaireet $R$.Brenot - R epères pour les D écisions en Chirurgie V asculaire. 2000:31-5.

3. Faries PL, Arora S, Pomposelli FB, et al. The use of arm vein in lower-extremity revascularization: results of 520 procedures performed in eight years. J V asc Surg. 2000;31:50-9.

4. Faries PL, LoG erfo FW, AroraS, et al. Arm vein is superior to composite prosthetic-autogenous grafts in lower-extremity revascularization. J V asc Surg. 2000;31:1119-27.

5. Chang BJ, D arling III RC, Bock D, Shah D M, Leather RP. The use of spliced vein bypasses for infrainguinal arterial reconstruction. J V asc Surg. 1995;21:403-12.

6. M ellière $D$, le C hevillier $B$, Kovarsky $S$. W rapped autologous greater saphenous vein bypass for severe limb ischemia in patients with varicose veins. Preliminary report. J Cardiovasc Surg. 1995;36:117-20.

7. Kunlin J, Bitry-Boely C, Pajot A. Evolution sur 28 ans d'un long pontage veineux fémoro-poplité pour artériopathie Actualités Chirurgicales - 79éme Congrès Français de Chirurgie. 1978, M ASSO N édit.

8. Cassina PC, H ailemariam SE, Schmid RA, et al. Infrainguinal aneurysm formation in arterialized saphenous vein grafts. J V asc Surg. 1988;28:944-8.

9. Peer RM , U pson JF. A neurysmal dilatation in saphenous vein bypass grafts. J Cardiovasc Surg. 1990;31:668-71.

10. L oftus IM , M CC arthy M J, Lloyd A, et al. Prevalence of true vein graftsaneurysms: implication for aneurysmspathogenesis. J V asc Surg. 1999;29:403-8.

11. CorriereM A, Passman M A, G uzman RJ , D attilo JB, N aslund TC. M ega-aneurysmal degeneration of a saphenous vein graft following infrainguinal bypass. A case report. V asc Endovasc Surg. 2004;38:267-71.

12. Kelly PH, Julsrud JM, Dyrud PE, Blake DP. Aneurysmal rupture of a femoropopliteal saphenous vein graft. Surgery. 1990;107:468-70.

13. Fukui $S, G$ oëau-Brissonière $O, C$ oggia $M, F$ ranc $P$, Patel JC. Rupture à la peau d'un Anévrisme athéromateux sur un Pontage Veineux femoro-poplité par veine in-situ. Presse médicale (Paris). 1996;25:1631-2.

14. D aviesM G, H agen PO . Pathophysiology of vein graftsfailures: a review. Eur J Endovasc Surg. 1995;9:7-18.

15. $M$ oritz $A$, Raderer $F, M$ agometschnigg $H$, et al. The use of $M$ esh-tube-constricted dilated or varicose vein as arterial bypass conduit: Thor C ardiovasc Surg. 1992;40:356-60.

16. Soury $P$, Peillon $C, W$ atelet J, et al. $M$ anchonnageprothétique des veines saphénes variqueuses dans les pontages sousinguinaux. Ann Chir Vasc. 1999;13:290-3.

17. N eufang A, D orweiler B, Espinola-Klein C, et al. External reinforcement of varicose veins with PTFE prosthesis in infrainguinal bypass surgery - clinical results. Thoracic C ardiovasc Surg. 2003;51:62-6.

18. Karayannacos $P E, H$ ostotler $J R, B$ ond $M G$. Late failure in vein grafts: mediating factorsin subendothelial fibromuscular hyperplasia. Ann Surg. 1978;187:183-8.

\section{Correspondência:}

Didier M ellière

Cirurgia Vascular

$\mathrm{CHU} \mathrm{H}$ enri $\mathrm{M}$ ondor

94010 Cretéil, França

E-mail: didier.melliere@laposte.net 